Original Article

\title{
Effects of running in place accompanied by abdominal drawing-in on the posture of healthy adults
}

\author{
Misuk Cho, $\mathrm{PhD}, \mathrm{PT}^{1)}$ \\ 1) Department of Physical Therapy, Korea Nazarene University: Wolbong Ro 48, Seobuk-gu, \\ Cheonan-Si, Chungcheongnam-do 330-718, Republic of Korea
}

\begin{abstract}
Purpose] This study aimed to determine the effect of running in place accompanied by abdominal drawing-in on the posture of healthy adults. [Subjects] A total of 30 subjects were divided into a training group and a control group, each containing 15 subjects. [Methods] The training group performed running in place accompanied by abdominal drawing-in for 30 min daily, three times a week for six weeks. Trunk inclination (TIN), trunk imbalance (TIM), pelvic position (PPO), pelvic torsion (PTO), pelvic rotation (PRO), and position of the scapulae (PSA) were assessed using BackMapper for both the training group and the control group before and after the experiment. [Results] Comparison of the results of the training and control groups before and after the experiment showed that the training group had statistically significant changes in TIN, TIM, PPO, PTO, and PSA, while the control group had no statistically significant changes in any items. [Conclusion] Running in place can be conveniently utilized by students or workers, regardless of time and place, as an exercise for postural improvement.

Key words: Running in place, Posture, BackMapper
\end{abstract}

(This article was submitted Jan. 13, 2015, and was accepted Jan. 31, 2015)

\section{INTRODUCTION}

Many dysfunctions are increasing in modern society due to excessive sedentary hours, which are a result of advancements of industrial society, and a lack of exercise. Excessively sedentary lifestyles cause weakening of the abdominal muscles and poor posture, as well as physical imbalance. Spinal deformity due to these factors can cause serious health problems and low back pain ${ }^{1)}$. In particular, excessive biomechanical burdens due to a sedentary lifestyle can induce weakening of abdominal muscles, general muscle weakening, instability of spinal joints, pain in the lumbar spine, reduced endurance, and restriction of flexibility and joint range of motion (ROM) $)^{2}$.

Abnormal posture can cause various problems, including flat-back syndrome, cervical kyphosis, and local organ ailments, such as uterine prolapse and gastric herniation ${ }^{3)}$. One of the main deep muscles for lumbar stability is the transversus abdominis ${ }^{4}$. A number of studies have reported the importance of the transversus abdominis, which contributes significantly to lumbar stability and posture ${ }^{5)}$.

To improve reduced lumbar stability caused by modern sedentary lifestyles, running in place while contracting the transverse abdominis and the multifidus through abdominal

Corresponding author. Misuk Cho (E-mail: mscho@kornu. ac.kr)

C2015 The Society of Physical Therapy Science. Published by IPEC Inc. This is an open-access article distributed under the terms of the Creative Commons Attribution Non-Commercial No Derivatives (by-ncnd) License $<$ http://creativecommons.org/licenses/by-nc-nd/3.0/> . drawing-in has been recommended. Exercises similar to running in place, such as plyometrics ${ }^{6}$, circuit training ${ }^{7)}$, and core stability exercises ${ }^{8}$, have been proposed. These exercises improve trunk muscle strength and flexibility because they strengthen the core muscles that together maintain lumbar stability ${ }^{9)}$. A number of studies on postural improvement can be found in the literature. These include lumbo-pelvic stabilization and postural improvement through bridge exercises $^{10)}$ and various mat exercises ${ }^{11)}$, lumbar stabilization improvement through running in place ${ }^{12)}$, and gait improvement ${ }^{13)}$.

However, plyometrics, circuit training, core stability exercises, bridge exercises, and mat exercises require systematic programs under the supervision of experts, as well as equipment and appropriate locations for the exercises. Because of these drawbacks, typical students and workers cannot employ such exercises for the purpose of postural correction. In contrast, running in place can conveniently be done regardless of time and location. Although studies on running in place have been conducted to report on lumbar stability improvement, none have been conducted on running in place to verify postural improvements.

Therefore, this study aimed to determine the effects or running in place, which can be done regardless of time and place and without the aid of tools or equipment, for six weeks on postural changes.

\section{SUBJECTS AND METHODS}

This study choose 30 university students and randomly divided them into a training group ( 2 males and 13 females) that participated in running in place and a control group (2 
males and 13 females) that did not run in place. The selection criteria were as follows: no structural abnormality of the spine before participation in the experiment, no pain in the spine (such as low back pain), no medication, no neurological diseases, no drinking before the experiment, and not overweight. Those who performed weight training, which can affect posture, and those who performed regular exercise were excluded from the experiment. This study was approved by university's institutional review board and the subjects were safely protected during all of the processes of the experiment. All of the subjects understood the purpose of this study and provided written informed consent prior to participation in the study in accordance with the ethical standards of the Declaration of Helsinki. The mean age, height, and weight of the training group were $22.6 \pm 0.3$ years, $161.5 \pm 3.1 \mathrm{~cm}$, and $56.3 \pm 6.5 \mathrm{~kg}$ respectively. The mean age, height, and weight of the control group were $21.8 \pm 0.6$ years, $162.5 \pm 5.3 \mathrm{~cm}$, and $56.2 \pm 5.3 \mathrm{~kg}$ respectively. Analysis of gender was done by $\chi^{2}$ test, and analysis of age, height, and weight was done by independent t-test. In the analysis, no statistically significant difference was found $(\mathrm{p}>0.05)$, so that no issues were found to be homogeneous between the two groups.

To maintain correct posture while running in place, a $30 \times$ $30-\mathrm{cm}$ square mark was placed on the floor and the subjects were positioned in its center. The subjects kept their waists straight and looked to the front by pulling in the chin, thereby maintaining the neutral position of the cervical spine. They contracted the transverse abdominis and multifidus muscles through abdominal drawing-in in order to maintain a neutral position of the waist and pelvis and ran in place in the limited square space without wobbling to the front, back, right, or left. To apply the principle of incremental loads and an appropriate exercise-strength setup, only two sets were performed during the first and second weeks out of a total of six weeks, and three sets were performed during the third to sixth weeks. Each set consisted of 20 running units followed by a 15 -sec rest, 20 running units followed by a 15 -sec rest, 30 running units followed by a 20 -sec rest, 30 running units followed by a 20 -sec rest, and then 30 running units. A 3-min rest was given between sets. One running unit was defined as the feet touching the ground one after the other. The speed of running in place was set to 17 running units per $10 \mathrm{sec}^{12)}$. A 5-min warm-up was conducted before the main exercise began and light stretching was conducted as a cooldown after the main exercise for $5 \mathrm{~min}$, resulting in $30 \mathrm{~min}$ of exercise three times a week for six weeks. The subjects in the control group performed their normal daily routines without any particular exercise and were subjected to two sets of measurements.

To measure posture, BackMapper (ABW, Frickenhausen, Germany), a three-dimensional-image spine diagnostic system, was used. This equipment can measure the shape and location of the spine, and the degree of twist in the pelvis relatively precisely, by viewing the spine from the front, back, lower, and upper sides, as well as in the sagittal plane. It can also analyze trunk inclination (TIN) in the sagittal plane, trunk imbalance (TIM) in the coronal plane, pelvic position (PPO), pelvic torsion (PTO), pelvic rotation (PRO) in the horizontal plane, and position of the scapulae (PSA)
Table 1. Comparison of TIN, TIM, PPO, PTO, PRO and PSA between before and after the experiment in each group (mean \pm SD) (unit: TIN, TIM, PPO, PTO and PRO, degrees; PSA, mm)

\begin{tabular}{|c|c|c|c|}
\hline Category & Group & Before & After \\
\hline \multirow{2}{*}{ TIN } & Training $\mathrm{G}^{*}$ & $2.5 \pm 1.3$ & $1.2 \pm 0.8$ \\
\hline & Control G & $2.5 \pm 0.9$ & $2.4 \pm 1.4$ \\
\hline \multirow{2}{*}{ TIM } & Training $\mathrm{G}^{*}$ & $2.5 \pm 2.4$ & $1.1 \pm 0.8$ \\
\hline & Control G & $2.5 \pm 1.5$ & $2.5 \pm 1.4$ \\
\hline \multirow{2}{*}{ PPO } & Training $\mathrm{G}^{*}$ & $2.4 \pm 1.2$ & $1.2 \pm 0.7$ \\
\hline & Control G & $2.4 \pm 1.4$ & $2.8 \pm 1.6$ \\
\hline \multirow{2}{*}{ PTO } & Training $\mathrm{G}^{*}$ & $3.6 \pm 2.4$ & $1.1 \pm 0.3$ \\
\hline & Control G & $3.2 \pm 2.9$ & $3.0 \pm 2.0$ \\
\hline \multirow[t]{2}{*}{ PRO } & Training G & $2.3 \pm 1.5$ & $1.5 \pm 0.7$ \\
\hline & Control G & $2.3 \pm 1.6$ & $2.29 \pm 1.53$ \\
\hline \multirow[t]{2}{*}{ PSA } & Training $\mathrm{G}^{*}$ & $4.6 \pm 2.7$ & $2.6 \pm 1.3$ \\
\hline & Control G & $4.7 \pm 2.9$ & $4.5 \pm 3.0$ \\
\hline
\end{tabular}

${ }^{*} \mathrm{p}<0.05$. TIN: trunk inclination; TIM: trunk imbalance; PPO: pelvic position; PTO: pelvic torsion; PRO: pelvic rotation; PSA: position of scapulae

by showing the muscles, fat distribution, position of the skeleton, and various other factors.

The measured data were analyzed with the SPSS $12.0 \mathrm{KO}$ statistical program, and the collected data are presented as means and standard deviations. The paired t-test was used as a significance test in each group before and after the experiments, and an independent t-test was used as a significance test for between-group differences. The significance level, $\alpha$, was set at 0.05 .

\section{RESULTS}

Comparison of the results between the training group and the control group before and after the experiment showed that the training group showed statistically significant changes in TIN, TIM, PPO, PTO, and PSA, while the control group showed no statistically significant changes in any items $(p<0.05)$ (Table 1). Comparison of the differences between the training and control groups before the experiment, after the experiment, and between the first and last measurements showed that none of the items were statistically significantly different before the experiment; however differences in TIN, TIM, PPO, PTO, and PSA showed statistical significance after the experiment, and TIM and PPO were statistically significantly different between the first and last measurements $(\mathrm{p}<0.05)$ (Table 2).

\section{DISCUSSION}

Correct posture is very important because it can affect not only the efficiency of bodily functions but also posture and outer appearance ${ }^{14)}$. Therefore, this study aimed to determine the effect of running in place on postural correction for normal adults. Running in place can reduce muscle fatigue and put loads on various muscles, thereby improving aerobic exercise ability and muscle strength at the same time, as well 
Table 2. Comparison of TIN, TIM, PPO, PTO, PRO and PSA between the training group and control group (mean $\pm \mathrm{SD}$ ) (unit: TIN, TIM, PPO, PTO and PRO, degrees; PSA, mm)

\begin{tabular}{lccc}
\hline & Category & Training G & Control G \\
\hline & TIN & $2.5 \pm 1.3$ & $2.5 \pm 0.9$ \\
& TIM & $2.5 \pm 2.4$ & $2.5 \pm 1.5$ \\
Before experiment & PPO & $2.4 \pm 1.2$ & $2.4 \pm 1.4$ \\
& PTO & $3.6 \pm 2.4$ & $3.2 \pm 2.9$ \\
& PRO & $2.3 \pm 1.5$ & $2.3 \pm 1.6$ \\
& PSA & $4.6 \pm 2.7$ & $4.7 \pm 2.9$ \\
& TIN & $1.2 \pm 0.8$ & $2.4 \pm 1.4$ \\
After experiment & TIM $^{*}$ & $1.1 \pm 0.8$ & $2.5 \pm 1.4$ \\
& PPO $^{*}$ & $1.2 \pm 0.7$ & $2.8 \pm 1.6$ \\
& PTO $^{*}$ & $1.1 \pm 0.3$ & $3.0 \pm 2.0$ \\
& PRO $^{*}$ & $1.5 \pm 0.7$ & $2.2 \pm 1.5$ \\
Change between & PSA & $2.6 \pm 1.3$ & $4.5 \pm 3.0$ \\
before and after & TIN & $1.2 \pm 1.4$ & $0.1 \pm 1.7$ \\
the experiment & TIM & $1.4 \pm 2.3$ & $0.0 \pm 1.4$ \\
& PPO & $1.1 \pm 1.5$ & $-0.4 \pm 1.7$ \\
& PTO & $2.4 \pm 2.5$ & $0.23 \pm 4.4$ \\
& PRO & $0.7 \pm 1.7$ & $0.0 \pm 2.3$ \\
& PSA & $2.0 \pm 2.6$ & $0.1 \pm 4.4$ \\
\hline
\end{tabular}

${ }^{*} \mathrm{p}<0.05$

as strengthening the trunk and abdominal muscles that contribute to lumbar stabilization ${ }^{12)}$. In addition, it can improve trunk muscle strength and flexibility and changes in posture by improving gait and lumbar stability due to the dynamic motion of the lower and upper body. Exercises similar to running in place, such as Pilates, core stabilization exercises, plyometrics, and circuit training have been previously studied. Endleman reported that performance of intensive Pilates that focused on the core by 18 healthy adult females and eight adult males for six months resulted in a significant difference in the transversus abdominis after measuring its muscle thickness and in the internal oblique abdominis, thereby proving the positive effect of core training on abdominal strength and trunk stabilization ${ }^{15)}$. Brill proposed that core stabilization exercises can maintain spinal balance based on contraction of the transverse abdominis, thereby increasing lumbar muscle strength and stability ${ }^{9}$. Myer proved that plyometrics and core stabilization exercises were effective in controlling the nerve roots and muscle power by having female high school athletes perform such exercises three times a week for seven weeks ${ }^{16)}$.

In a study on postural changes, Cho placed 15 female university students into a pelvic adjustment group and $15 \mathrm{fe}-$ male university students into a stretching group and then performed pelvic adjustment through the Gonstead technique, which had a positive effect on postural changes ${ }^{14)}$. In a study on running in place, Cho and Jun divided 30 participants into a training group and a control group, each containing 15 subjects; the training group performed running in place for six weeks and the results showed improved dynamic lumbar stability ${ }^{12)}$. Cho reported that running in place can improve step length difference, swing phase difference, single support difference, and step time difference ${ }^{13)}$.

Comparison of the results of the training group and the control group before and after the experiment in the present study showed that the training group had statistically significant changes in TIN, TIM, PPO, PTO, and PSA, while the control group had no statistically significant changes in any items. These results showed that running in place can improve mobility of the waist and pelvis, which are related to posture. They also showed that positive changes in posture can occur as a result of running in place, accompanied by dynamic movements in the upper and lower extremities, in a square-shape space with no wobbling while maintaining muscle contraction of the transversus abdominis and applying the abdominal drawing-in method. Although various exercise methods for postural correction have been proposed previously, the methods required systematic programs under the supervision of experts, as well as equipment and appropriate locations for the exercises. Therefore, we expect that running in place can be utilized as an exercise for postural correction by students or workers in the clinical field who are restricted by time and place.

This research was supported by Korea Nazarene University Research Grants in 2015.

\section{ACKNOWLEDGEMENT}

This research was supported by Korea Nazarene University Research Grants in 2015.

\section{REFERENCES}

1) Bae JS, Jang JS, Lee SH, et al.: A comparison study on the change in lumbar lordosis when standing, sitting on a chair, and sitting on the floor in normal individuals. J Korean Neurosurg Soc, 2012, 51: 20-23. [Medline] [CrossRef]

2) Gill K, Krag MH, Johnson GB, et al.: Repeatability of four clinical methods for assessment of lumbar spinal motion. Spine, 1988, 13: 50-53. [Medline] [CrossRef]

3) Tsuji T, Matsuyama Y, Goto M, et al.: Knee-spine syndrome: correlation between sacral inclination and patellofemoral joint pain. J Orthop Sci, 2002, 7: 519-523. [Medline] [CrossRef]

4) Gong W: Correlations between transversus abdominis thickness, lumbar stability, and balance of female university students. J Phys Ther Sci, 2013, 25: 681-683. [Medline] [CrossRef]

5) Hodges PW: Is there a role for transversus abdominis in lumbo-pelvic stability? Man Ther, 1999, 4: 74-86. [Medline] [CrossRef]

6) Kraemer WJ, Mazzetti SA, Nindl BC, et al.: Effect of resistance training on women's strength/power and occupational performances. Med Sci Sports Exerc, 2001, 33: 1011-1025. [Medline] [CrossRef]

7) Williams PA, Cash TF: Effects of a circuit weight training program on the body images of college students. Int J Eat Disord, 2001, 30: 75-82. [Medline] [CrossRef]

8) Saliba SA, Croy T, Guthrie R, et al.: Differences in transverse abdominis activation with stable and unstable bridging exercises in individuals with low back pain. N Am J Sports Phys Ther, 2010, 5: 63-73. [Medline]

9) Brill PW, Suffes S, Witz M, et al.: The Core Program: Fifteen Minutes a Day that Can Change Your Life. New York: Buntam Books. 2008, 1-231.

10) García-Vaquero MP, Moreside JM, Brontons-Gil E, et al.: Trunk muscle activation during stabilization exercises with single and double leg support. J Electromyogr Kinesiol, 2012, 22: 398-406. [Medline] [CrossRef]

11) Liebenson $C$ : Spinal stabilization training: the transverse abdominus. J Bodyw Mov Ther, 1998, 2: 218-223. [CrossRef]

12) Cho M, Jun I: The effects of running in place on healthy adults' lumbar stability. J Phys Ther Sci, 2014, 26: 821-824. [Medline] [CrossRef]

13) Cho M: Effects of running in place accompanied by abdominal drawing-in on gait characteristics of healthy adults. J Phys Ther Sci, 2015, 27: 87-89. 
1616 J. Phys. Ther. Sci. Vol. 27, No. 5, 2015

[Medline] [CrossRef]

14) Cho M: The influence of pelvic adjustment on the posture of female university students. J Phys Ther Sci, 2013, 25: 785-787. [Medline] [CrossRef]

15) Endleman I, Critchley DJ: Transversus abdominis and obliquus internus activity during pilates exercises: measurement with ultrasound scanning.
Arch Phys Med Rehabil, 2008, 89: 2205-2212. [Medline] [CrossRef]

16) Myer GD, Ford KR, Brent JL, et al.: The effects of plyometric vs. dynamic stabilization and balance training on power, balance, and landing force in female athletes. J Strength Cond Res, 2006, 20: 345-353. [Medline] 